MATEC Web of Conferences 22,02004 (2015)

DOI: $10.1051 /$ matecconf/ 20152202004

(C) Owned by the authors, published by EDP Sciences, 2015

\title{
Active Power Control Simulation Platform Research of Wind Farm Based on Multi-Agent
}

\author{
Xingiie Liu \& Kangyuan Wang \\ Electrical and Electronic Engineering Institute, North China Electric Power University, Baoding, Hebei, China
}

\begin{abstract}
The realization of the automation, routinization and intelligentization of dispatch control in wind farms is the key to the integration of wind farms into power grid management system. Active power regulate and control system in wind farms has increasingly high demand on timeliness, but at present this system is mostly equipped with centralized unidirectional control with poor timeliness and low utilization ratio for wind energy resources. The characteristics of distribution and instantaneity owned by the active power regulate and control system in wind farms are highly consistent with Multi-Agent system. This paper discusses a kind of processing method that is used in real-time, distributed and parallel computation and processing for multiple simultaneously running wind turbines, which is based on Multi-Agent technology and adopting JADE development platform. This method converts massive centralized computation to distributed computation, which optimizes the effect of the power control. This method makes the effectiveness of active power regulate and control system better, wins time for timely allocating electricity generation assignments and dealing with problems, and avoids the heavy loss of resources.
\end{abstract}

Keywords: Multi-Agent system; active power; optimized regulate and control; instantaneity; distribution

\section{INTRODUCTION}

At present, wind energy is regarded as a kind of clean, safe and renewable green energy, receiving increasing attention from all over the world. And wind power generation is one of the most important links. As the technology of wind power generation matures and the installed wind power capacity constantly expands, the position of wind power generation has become increasingly prominent in power grid. However, wind energy has intermittency, volatility, anti-peak load regulation and other characteristics. When the wind energy is accessed to large-scale power grids, unstable wind energy is bound to have an impact on power balance, frequency stability and other factors involved safe and economic operation of power grids. In order to ensure the safety of wind power connected to power grids, wind abandoning measures are usually adopted, which results in the waste of large amounts of wind energy[1]. Effectively controlling the active power of wind farms can lower the impact of randomness and volatility of wind power on power grids. Under the present conditions of power grids, its acceptance capacity for wind power shall be improved to achieve energy conservation and emission reduction. Therefore, it's important and theoretically and practically valuable to carry out regulate and research for active power in wind farms and develop more advanced and intelligent active power regulate and control system in wind farm.

Wind farms are generally composed of numerous wind turbine generators distributed in tens to hundreds of square kilometers and are a distributed, complex and high-dimensional large-scale system. When establishing relevant active power control model, the number of variables and constraints may be up to several hundred, and their online calculation and implementation method are the issues that need key consideration. A lot of researches on active power regulate and control in wind farms have been made from literature [2] to literature [7]. But many ideas they express on how wind farms respond to power grid dispatch in instantaneity and other problems are not sufficient. The automation, routinization and intelligence of dispatch and control in wind farms cannot be achieved, and it's unable to make wind farms have the similar adjustment capability to traditional energy power plants. Whereas, having the above features and adjustment capability is the key to fitting into an existing power grid management system.

At this stage, due to large and complex problems to be optimized, the amount of computation for overall centralized solution method will be incomparably huge, which will generate excessive economic costs, and the efficiency of instantaneity optimization is lower. It is an effective way to solve these problems by using distributed solution algorithm. Furthermore, in view of the advantages of solving distributed problems through Multi-Agent technology, this paper recommends to adopt Multi-Agent technology to construct an active power regulate and control system in wind farms, to lead the principle and the technology of Agent and Multi-Agent system into regulate and control decision of active power of wind turbine and to study new intellectualized control methods for the active power of wind farms. 


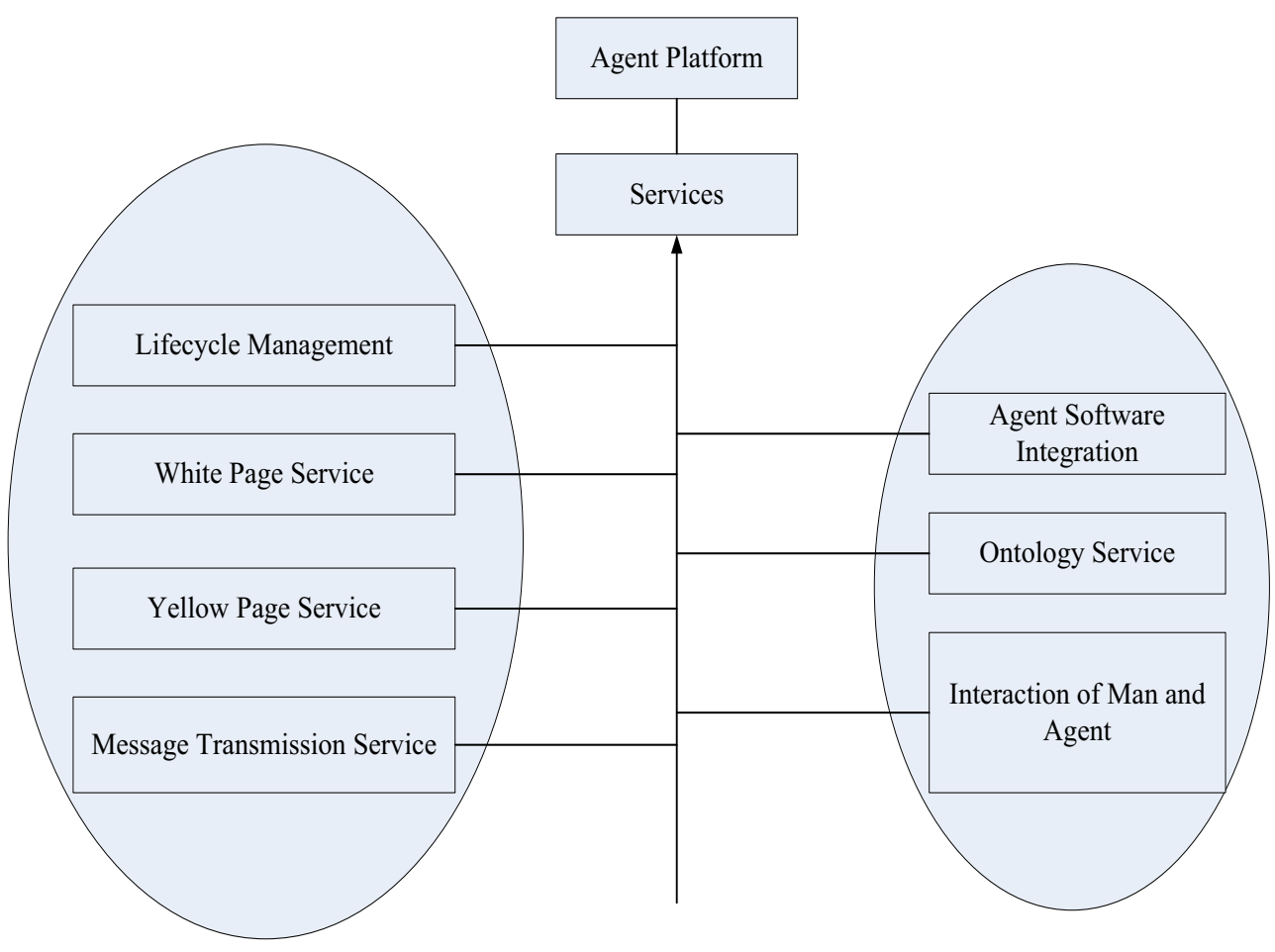

Figure 1. Services provided by JADE platform

Agent originates in distributed artificial intelligence, is the technology that combines artificial intelligence and distributed computation, may be seen as an entity that has autonomous capability, and can continuously and spontaneously achieve the design functions in a particular environment. Agent has the basic characteristics of autonomy, social nature, response capability, spontaneous behavior and so on ${ }^{[8]}$.

Multi-Agent system refers to a looser Multi-Agent union that consists of multiple interacted and interconnected Agents. These Agent members cooperate and serve mutually to complete an assignment together, and their collaborating and solving capabilities have been more than one single Agent ${ }^{[9]}$. The activity of each Agent member is autonomous and independent. Their own goals and behaviors are not restricted by other Agent members. The contradictions and conflicts between the goals and the behaviors of each Agent member are coordinated and resolved by means of competitions, negotiations or other methods.

The data and resources in Multi-Agent system are dispersed. Each Agent has incomprehensive information or capability for the assignments to be completed, and its concept of performing assignments is local. There is no global control system. The solution and calculation of the questions are conducted asynchronously. Multi-Agent system takes human society as research model. System science, decision science, management and organization theory, economics, game theory and other subjects, which are related to the study of social behaviors of human beings, provide a theoretical basis for the Multi-Agent system. The past, closed and isolated knowledge system will be developed into an open, distributed and intelligent knowledge system. Multi-Agent system not only has the advantage of solving distributed and concurrent problems, but also is widely used in modern power system. A paper [10] describes the use of parallel work and collaboration mechanisms in Multi-Agent system, and the construction of the architecture and work mechanism of Multi-Agent for wide area protection may effectively achieve fast adaptive protection for large-scale power grid system under different operating conditions. Another paper[11] uses dispersion, coordination, control and other advantages in Multi-Agent system, establishes distributed intelligent dispatch system to realize energy-saving power generation, and verifies that this dispatch system is more adaptive, intelligent and faster.

JADE (Java Agent Development Framework) is an open source development framework with Multi-Agent system that has been developed entirely by the java language. JADE follows FIPA specification, through a series of abstract interfaces and tools, and provides basic services and communications, so that the developers can skip the realization of the FIPA standards and can pay more attention to the realization of the functions in Multi-Agent system. JADE completely refers to the definition of FIPA to provide a number of services, which are shown in Figure 1. 


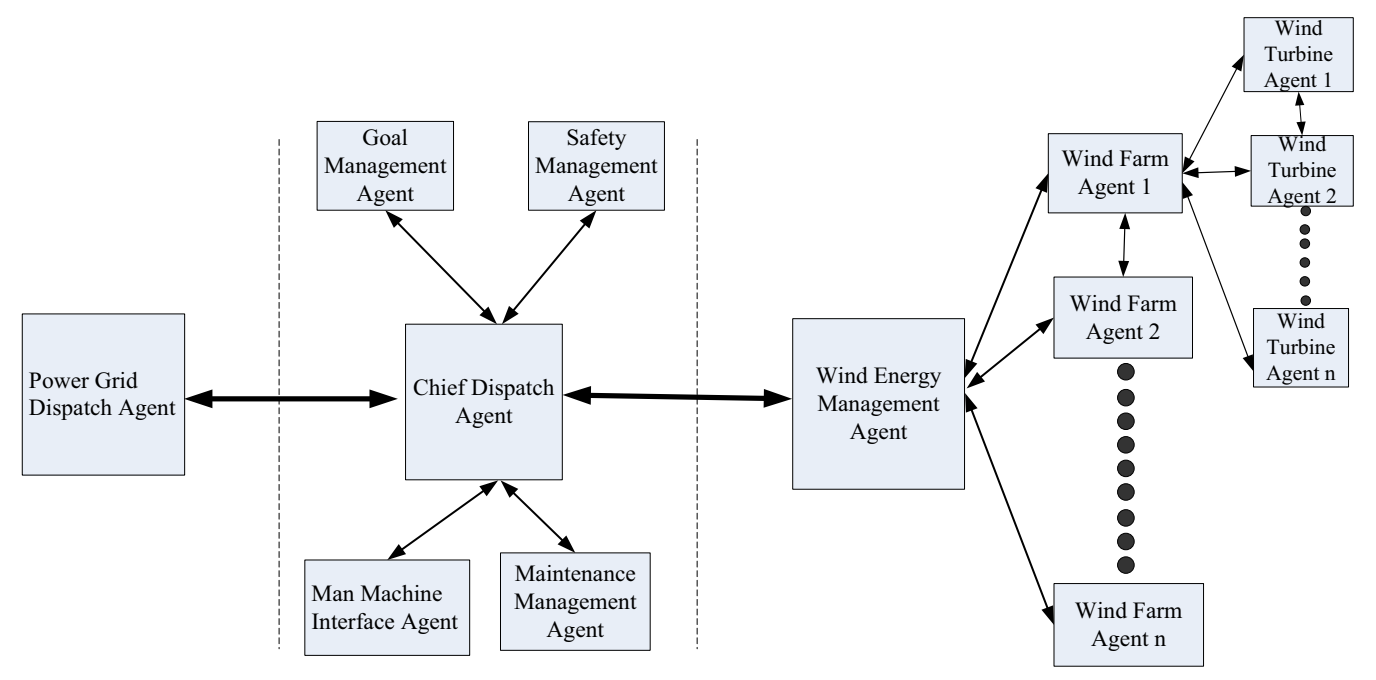

Figure 2. The structure of Multi-Agent system

\section{ACTIVE POWER REGULATE \& CONTROL SYSTEM IN WIND FARMS BASED ON MUL- TI-AGENT SYSTEM}

\subsection{Design of Agent}

This section applies the thought of Multi-Agent system structure and constructs an active power regulate and control system in wind farms based on Multi-Agent system. The architecture of the system is shown in Figure 2.

The composite architecture is used by this system and a variety of Agents have been designed. When the power grid issues a power command, the data is transmitted to the Chief Dispatch Agent through the Power Grid Dispatch Agent. The Chief Dispatch Agent carries out the process of power allocation, and eventually transmits the specific goals of power allocation are transmitted to Wind Turbine Agent that is the subordinate of each Wind Farm Agent through the Wind Energy Management Agent for the purpose of optimization, regulate and control of the power.

The duties and functions for each Agent are different. The specific instructions are as follows:

(1) Power Grid Dispatch Agent: accepts and conveys the instructions on power deployment from superior power grid, including starts and stops, cutting-off and so on. Power Grid Dispatch Agent is responsible for processing the instructions issued by the superior power grid and it cooperates with the Chief Dispatch Agent to optimize the system.

(2) Chief Dispatch Agent: this Agent is responsible for providing the decisions and instructions from power grid dispatch, and is responsible for the adjustment of power generating units, load allocation, the adjustment of generator output, the maintenance for generating units, peak regulation, frequency modulation and the arrangement for spare capacity.
(3) Wind Energy Management Agent: according to the resources available of wind turbine, this Agent determines upper and lower processing limits of generating units, and adjusts the output of the Wind Turbine Agent by mutual agreement with the Wind Farm Agent and its subordinate Wind Turbine Agent.

(4) Wind Turbine Agent and Wind Farm Agent: a wind turbine is regarded as a Wind Turbine Agent; a number of wind turbines connected by a bus bar can be classified as a Wind Farm Agent; numerous Wind Turbine Agents owned by the Wind Farm Agent are subject to the management of this Wind Farm Agent.

(5) Goal Management Agent: compared with the goal of traditional dispatch, the goal of intelligent dispatch not only considers economy, but also comprehensively and harmoniously considers economy, energy saving, environmental protection, safety and reliability.

(6) Safety Management Agent: the Agent not only will provide safe and stable constraints for the chief dispatch, but also can independently finish a stable verification task.

(7) Maintenance Management Agent: this Agent mainly determines the wind turbines that can be arranged for maintenance. Power Dispatch Agents at all levels should be based on the results of load forecasting and sorted table, on the premise of ensuring the safe operation of the system, comprehensively consider various factors, optimize the preparation of the yearly and monthly maintenance plan of generating units; according to the results of short-term load forecasting, arrange daily maintenance.

(8) Man-machine Interface Agent: Man-machine Interface Agent is responsible for the management and maintenance of man-machine interface in the entire system, which is user-oriented and able to display parameter tables and variation curve of system operation to the users, and the users can delete Agent, in- 
MATEC Web of Conferences

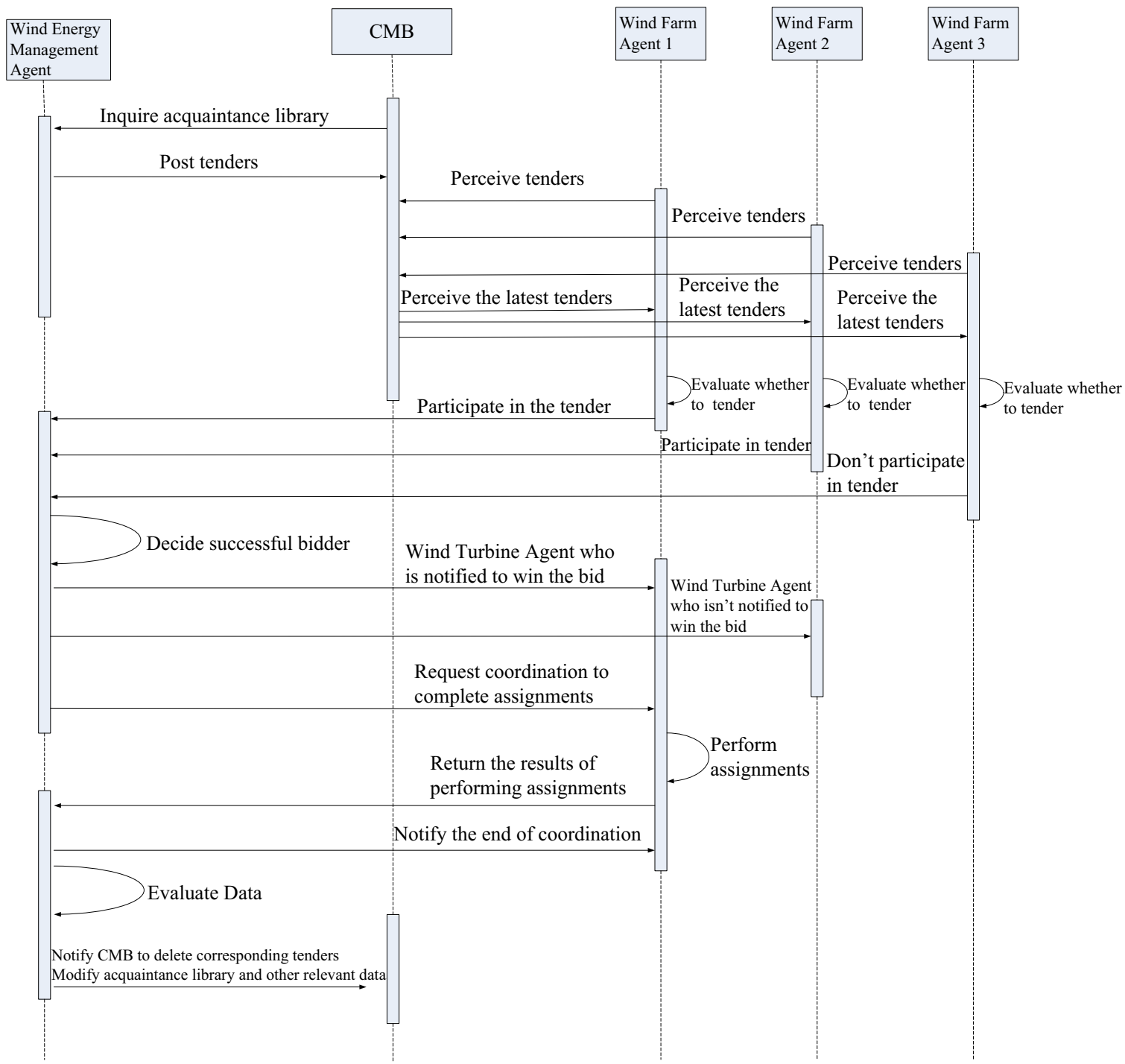

Figure 3. Algorithm block diagram of comprehensive contract net

crease Agent, edit Agent, and modify the setting of all parameters through the man-machine interface.

\subsection{Communication between Agents}

The communication between the Agents in JADE is based on asynchronous communication mode. The Sender Agent uses ACL Message object to send messages, and the object contains the unique name of the Receiver Agent, the sending content and other information. Each JADE Agent has a mailbox, that is, Agent Message Queues. Each Message Receiving Agent is equipped with the mailbox for receiving messages. After the message is conveyed, the Agent automatically invokes method action() in Cyclic Behaviour to respond to the message.

\subsection{Coordination algorithm between Agents}

This section proposes a coordination algorithm based on a comprehensive contract net between Agents. Through registering all levels of Agents, and selecting bid objects and other steps, we complete the delivery of information between each other, and realize mutual coordination and consultation between Agents. The block diagram of coordination algorithm is shown in Figure 3 .

Step 1: Registry of all levels of Agents

All Wind Turbine Agents are registered in their respective wind farms; all Wind Farm Agents are registered in Wind Energy Management Agents; Wind Energy Management Agent, Goal Management Agent and other Agents are registered in Chief Dispatch Agent. 
Step 2: Select bidding objects

First, Wind Energy Management Agent finds from the Wind Turbine Agents owned by each Wind Farm Agent in their own acquaintance libraries, then, unites all acquaintances and asks them whether they can complete the latest power regulate assignments issued by Chief Dispatch Agent. If the acquaintances may complete the assignments, directly send the message on requesting coordination; if the capability of acquaintances is insufficient, the Wind Energy Management Agent invites tenders from all Wind Farm Agents.

Step 3: Invite tenders

Chief Dispatch Agent issues assignments. Wind Energy Management Agent divides the adjusted data of active power issued by Chief Dispatch Agent to form tenders (bidding documents) according to the previous round of output proportion of each Wind Farm Agent. After that, several tenders are posted to a public message board (Common Message Board, CMB). All Wind Farm Agents periodically take the initiative to perceive the tenders from CMB. The Wind Turbine Agents owned by each Wind Farm Agent, according to the changes of wind speed, humidity and other environmental factors, determine their own maximum output power and input the results into their respective Wind Farm Agents. Wind Farm Agents depend on the results to calculate their own capability and decide whether to submit a tender.

The format of the tender:

Tender $=\{$ tenderID, WindManager, prohibit,

TaskDescription, Grade,TaskRestriction, EndTime

tenderID: represents ID of the tender, is the only identity uniformly allocated by CMB;

WindManager : represents the identity of Wind Energy Management Agent who initiates collaboration;

Prohibit: represents a collection of Wind Turbine Agents that are prohibited to bid under maintenance and other states;

TaskDescription: represents a simple description for the assignments;

Grade: represents the current level of the assignments;

TaskRestriction: represents that when Wind Farm Agent invites a tender, it must provide the constraint information, which is composed of the following factors:

TaskRestriction $=\{$ Time, Reliance, Quality, Perception $\}$

Time: represents the time required by the Wind Farm Agent to complete this assignment;

Reliance: represents the reliability of Wind Farm Agent for Wind Energy Management Agent;

Quality: represents the completion degree that Wind Farm Agent promises to complete assignments;

Perception: represents the degree that Wind Farm Agent perceives the outside world, which is perception coefficient, which is determined by the following factors:

$$
\text { Perception }=\{\text { Idle, } \text { Resource }\}
$$

Idle: represents the busy or idle degree of Wind Farm Agent

Resource: represents its own resources available of Wind Farm Agent;

EndTin: represents the deadline of submitting a tender

Step 4: Submit bids

Wind Farm Agent submits bids for the assignments according to loading condition, resource usage, and the degree of conformity to assignment constraints for its subordinate Wind Turbine Agent. The bidding strategy is as follows:

$\operatorname{Bid}(m, i, t)=\lambda_{1} \cdot \operatorname{Per}(m, i, t)+\lambda_{2} \cdot \operatorname{Cap}(m, i, t)+\lambda_{3} \cdot \operatorname{Re} l(m, i, t)$

$+\lambda_{4} \cdot \operatorname{Act}(m, i, t)+\lambda_{5} \cdot \operatorname{Fri}(m, i, t)$

Wherein:

$\operatorname{Bid}(m, i, t)$ : represents the expected value of Wind Turbine Agent $_{i}$ submitting bids for assignment $t$,

$\operatorname{Per}(m, i, t)$ : represents perception coefficient of Wind Farm Agent $_{i}$ for the tender, which is usually decided by the idle degree of Wind Farm Agent $_{i}$ and its own resources. The perception coefficient of Wind Farm Agent $_{i}$ may be expressed as:

$$
\operatorname{Per}(m, i, t)=C_{1} \cdot \operatorname{Idle}(m, i, t)+C_{2} \cdot R(m, i, t)
$$

Wherein:

Idle $(m, i, t)$ : represents the idle degree of Wind Farm Agent $_{i}$; comprehensively consider the operational conditions of all its subordinate Wind Turbines and measure the idle degree of its own assignments;

$R(m, i, t)$ : represents the resources available owned by Wind Farm Agent $_{i}$; comprehensively consider instantaneous wind speed information of all wind turbines and predict the information of wind speed;

$C_{1}$ and $C_{2}$ represents respective weights of the idle degree and available resources.

$\operatorname{Cap}(m, i, t)$ : represents its capability that Wind Farm Agent $_{i}$ completes promised assignments;

$\operatorname{Re} l(m, i, t)$ : represents the reliance that Wind Farm Agent ${ }_{i}$ completes the assignments given by Wind Energy

Management Agent $_{m}$

$\operatorname{Act}(m, i, t)$ : represents the activity degree of Wind Farm Agent $_{i}$. Wind Energy Management Agent $_{m}$ issues $t$ type assignments. The ratio of total number of bidding times of Wind Farm Agent $_{i}$ and total number of bidding times of all Wind Farm Agents is called the activity degree of Wind Farm Agent ${ }_{i}$ in all Wind Farm Agents on $t$ type assignments. Its expression is as follows:

$$
\operatorname{Act}(m, i, t)=N_{i}^{t} / \sum_{i=1}^{n} N_{i}^{t}
$$

Wherein:

$N_{i}^{t}$ : represents $t$ type assignments issued by Wind Energy Management Agent $_{m}$, total number of bidding times of Wind Farm Agent ${ }_{i}$

$$
\sum_{i=1}^{n} N_{i}^{t}: \text { represents } t \text { type assignments issued by }
$$

Wind Energy Management Agent $_{m}$, total number of 


\section{MATEC Web of Conferences}

bidding times of all Wind Farm Agents

$\operatorname{Fri}(m, i, t)$ : represents the friendship of Wind Farm Agent

$\operatorname{Fri}(m, i, t)=N_{m i}^{t} / N_{m}^{t}$

Wherein:

$N_{m i}^{t}$ : represents the number of times of Wind Farm

Agent $_{i}$ completing $t$ type assignments given by Wind Energy Management Agent

$N_{m}^{t}$ : represents the total number of times that Wind

Energy Management Agent $_{m}$ giving $t$

type assignments

$\lambda_{1}, \lambda_{2}, \lambda_{3}, \lambda_{4}, \lambda_{5}$ represent the weight values of perception, capability, reliance, activity degree and friendship, and $\lambda_{1}+\lambda_{2}+\lambda_{3}+\lambda_{4}+\lambda_{5}=1$

The idle degree of Wind Farm Agent $_{i}$ may be divided into three grades: busy, medium and idle. The perceptions of these three grades are respectively 1,2 , 3 . The less its own resources are and the busier the grade is, the lower its perception is; instead, the higher its perception is. Comprehensively consider above factors and calculate their respective expected value for the bid. If the expected value for the bid calculated by Wind Farm Agent $_{i}$ itself is over the specified threshold value, the bid will be submitted; if not, the bid will not be submitted.

Step 5: Win the bid

According to bidding information provided by many bidders, Wind Energy Management Agent $_{m}$ selects several bidders who can be united by Wind Energy Management Agent $_{m}$ to form a Agent group and sends a contract to each Wind Farm Agent who wins the bid for confirmation. But generally there are multiple satisfying Wind Farm Agent $_{i}$ in the system, and there are more than one Agent with the highest reliance in the system. So Wind Energy Management Agent $_{m}$ introduces an evaluation function to confirm these bidders, and Wind Energy Management Agent ${ }_{m}$ revises the bidding value of Wind Farm Agent through an evaluation function, which is as follows:

$M(i, t)=q \cdot(T-$ time $)+L \cdot \operatorname{Re} l(m, i, t)$

$+(1-L) \cdot \operatorname{Per}(m, i, t)$

Wherein:

$T$ : represents the time that Wind Energy Management Agent $_{m}$ asks to complete the assignments; time : represent the time that Wind Farm Agent promises to complete the assignments; $\operatorname{Re} l(m, i, t)$ : represent the reliance; $\operatorname{Per}(m, i, t)$ : represent perception; L: represents the weight of reliance in bidding and decision process.

Step 6: Sign contracts

Select and determine the optimal group of Wind Farm Agent ; after receiving the contract confirmation request send by Wind Energy Management Agent $_{m}$, the Wind Farm Agent decides whether to sign contracts with Wind Energy Management Agent $_{m}$ according to the idle degree and its own resource usage at the moment.

Step 7: Evaluation

After Wind Farm Agent $_{i}$ signs contracts with
Wind Energy Management Agent $_{m}$, Wind Farm Agent starts to perform the assignments.

If Wind Farm Agent ${ }_{i}$ can complete the assignments, the results of performing the assignments will be returned to Wind Energy Management Agent $_{m}$, its own capability and reliance of this Wind Farm Agent ${ }_{i}$ will be modified. The expressions are as follows:

$$
\begin{aligned}
& \operatorname{Cap}(m, i, t)=\min [\operatorname{Cap}(m, i, t)+\Delta c, 1] \\
& \operatorname{Re} l(m, i, t)=\min [\operatorname{Re} l(m, i, t)+\Delta t, 1]
\end{aligned}
$$

Wherein:

$\Delta c$ : represents the increased value in its own capability; $\Delta t$ : represent the increased value in reliance

If the assignments cannot be completed successfully, likewise, its own capability and reliance will be modified.

Step 8: Notify CMB and modify its own database

Through Wind Energy Management Agent $_{m}$ notifying $\mathrm{CMB}$, modify the corresponding completed tenders and store the obtained data from evaluation into $\mathrm{CMB}$ for the next bidding purposes. At this point, a round of assignments is completed.

\section{ANALYSIS OF NUMERICAL EXAMPLES}

In this paper, four wind turbines and six time periods of wind power generation system are taken as research background. The system parameters are shown in Table 1, in which there are four Wind Turbine Agents $p_{i}$ represents the rated output power of the wind turbines; $p_{i 0}$ represents the actual output power of the wind turbine $i$ at the initial stage. System loads in each time period are shown in Table 2 .

Table 1. System parameters

\begin{tabular}{|l|l|l|l|l|}
\hline Name & $\mathrm{A} 1$ & $\mathrm{~A} 2$ & $\mathrm{~A} 3$ & $\mathrm{~A} 4$ \\
\hline$p_{i}$ & 20 & 16 & 17 & 15 \\
\hline$p_{i 0}$ & 16 & 7 & 8 & 8 \\
\hline
\end{tabular}

Table 2. Load data

\begin{tabular}{|l|l||l|l||l|l|}
\hline $\begin{array}{l}\text { Time } \\
\text { Period }\end{array}$ & Load & $\begin{array}{l}\text { Time } \\
\text { Period }\end{array}$ & Load & $\begin{array}{l}\text { Time } \\
\text { Period }\end{array}$ & Load \\
\hline 1 & 24 & 3 & 31 & 5 & 38 \\
\hline 2 & 25 & 4 & 37 & 6 & 41 \\
\hline
\end{tabular}

It is assumed that the actual power of the wind turbine is generally less than its rated power in the six time periods. Using the above coordination algorithm of the comprehensive contract net, we can get the optimal power allocation scheme of the four wind turbines. Actual outputs of each Agent in different time periods are shown in Table 3. As can be seen from Table 3, the proposed structure and optimization method for Multi-Agent System based on comprehensive contract net in this paper, may coordinate and optimize several wind turbines in a wind farm, at the same time of ensuring output, may utilize wind power resources to the greatest extent and extend the life of the wind turbine. 
ICETA 2015

Table 3. Output in different time periods

\begin{tabular}{|l|l|l|l|l|}
\hline Time Period & A1 & A2 & A3 & A4 \\
\hline 1 & 10 & 8 & 3 & 3 \\
\hline 2 & 10 & 8 & 4 & 3 \\
\hline 3 & 12 & 9 & 6 & 4 \\
\hline 4 & 13 & 11 & 8 & 5 \\
\hline 5 & 13 & 11 & 8 & 6 \\
\hline 6 & 14 & 12 & 8 & 7 \\
\hline
\end{tabular}

\section{CONCLUSION}

This paper is based on JADE development platform, takes full use of the advantages of intelligently decentralized coordination and control in Multi-Agent system, and establishes Multi-Agent system model of active power regulate and control in wind farms; when the operating condition changes, each Agent can perceive the changes of the conditions in the external environment, gain the knowledge and data required by mutual coordination and consultation, and start reasoning and resolver to achieve the desired objectives in accordance with the relevant rules; at the same time, adopt coordination algorithm of comprehensive contract net and utilize numerical examples for simulation to reach the explaining effect. Each Agent is mutually independent to achieve the effect of working in parallel, thus which greatly reduces the time of dispatching decision, meets the goals and requirements of the maximum use of wind energy in wind farms and achieves the optimized control of active power in wind farms.

\section{ACKNOWLEDGEMENT}

This work was supported by the Natural Science Foundation of Hebei Province of China (No. E2015502066).

\section{REFERENCES}

[1] Lei, Y.Z. 2003. Research topic related to grid connection of wind power. Automation of Electric Power Systems, 27 (8): 84-89.

[2] Xu, S.Y. \& Zhang, D.Y. 2011. Study on active power control in DFIG-based wind farm. IEEE Power Engineering and Automation Conference, 11: 192-195.

[3] Luo, C.L., Huadi Banakar. \& Bai, K.S.,et al. 2007. Strategies to smooth wind power fluctuations of wind turbine generator. IEEE transactions on energy conversion, $\mathrm{v} 22(2): 341-349$.

[4] Le, R., Chang, C.E. \& Yao, C.Y. 2009. Strategies for operating wind farm in a similar manner of conventional power plant. IEEE Transaction on Energy Conversion, V 24(4): 926-934.

[5] Calos F Moyano. \& Joao A Pecas Lopes. 2009. An optimization approach for wind turbines commitment and dispatch in a wind park. Electric power systems research, 79: 71-79.
[6] Yin, Y., Lu, J. P. \& Liu, G., et al. 2012. Active power distribution optimization of wind farm based on rotational kinetic energy of DFIG. Power System Protection and Control, 40(17): 127-132.

[7] Xiang, L., Xie, Y. \& Zhang, B., et al. 2013. Dynamic grouping strategy for active power control of wind farm $[\mathrm{C}] / /$ Cyber Technology in Automation, Control and Intelligent Systems (CYBER), 2013 IEEE 3rd Annual International Conference on. IEEE, pp: 132-137.

[8] Shi, Z.Z. 1998. Advanced Artificial Intelligence, Beijing: Science Press.

[9] Durfee E H, Lesser V R. \& Corkill D D. 1989. Trends in cooperative distributed problem solving. Knowledge and Data Engineering, IEEE Transactions on, 1(1): 63-83.

[10]Li, Z.X., Yin, X.G. \& Zhang, Z. 2012. Research based on Multi-Agent wide area protection system architecture. Power System Protection and Control, 40 (4): 71-75.

[11]Li, J.H., Wei, H. \& Xia, X.Q. 2010. The study on Multi-Agent system of energy conservation power generation dispatch under smart power grids. Power System Protection and Control, 38 (21): 96-103, 108. 